\title{
Mean shear stress effect built into the multiaxial fatigue strength estimation method of an integral type
}

\author{
Jan Papuga ${ }^{1,1}$, Lukáš Suchý ${ }^{2}$ Milan Růžička ${ }^{1}$ \\ ${ }^{1}$ FME, Czech Technical University in Prague, Technická 4, 16607 Prague 6, Czech Republic \\ ${ }^{2}$ Institute of Design Engineering and Drive Technology, Technische Universität Chemnitz, 09126 \\ Chemnitz, Germany
}

\begin{abstract}
The effect of mean shear stress is implemented into the PIR criterion [1-2]. Results of the new solution are only slightly worse than the similarly improved PCN criterion described in [3]. Five methods of the integral type known to the author (PIR + new PIN, the Fogue method, the Liu \& Zenner method, the Papadopoulos method) are evaluated here on a large set of experimental data (286 items). Because the integral solution is easier to handle, the simplified reduced formulas of each criterion for the case of purely axial loading are presented. Their relations each to other, and also to other common uniaxial mean stress effect methods are then studied and small numerical benchmark convening this load condition is prepared.
\end{abstract}

\section{Introduction}

Papuga and Halama [3] showed recently, how involvement of the mean shear stress parameter in a multiaxial fatigue strength estimation criterion can affect its prediction quality, including a substantially better response of the method even for purely axial load cases. This proof concerned the PCN criterion, which belongs among critical plane criteria maximizing the value of the damage parameter evaluated on each plane.

In this paper, the effect of the mean shear stress is implemented into the PIR criterion of the author [1-2]. This is a criterion of an integral type, i.e. of the damage parameter evaluated on each plane and integrated over all possible plane orientations. The paper of Tomčala et al. [4] discusses the effect of the step between individual orientations of evaluated planes, and it concludes that the integral solution is less sensitive to the reduction of the number of evaluated planes in comparison with the critical plane solution (approximately one half of planes is necessary to reach the same discretization error). Such conclusion makes the criteria of the integral type much more interesting for a future industrial use. Today, the leading fatigue solvers tend to focus solely on the multiaxial fatigue strength estimation based on the critical plane evaluation.

Five methods of the integral type known to the author (PIR + new PIN, the Fogue method, the Liu-Zenner method, the Papadopoulos method) are evaluated here for the complete set of

Corresponding author: papuga@ pragtic.com, jan.papuga@ fs.cvut.cz 
experimental data base (286 items). This data set is a full version of the benchmark, synopsis of which is presented in [5]. The differences between individual methods are commented in the discussion. This commentary is based on an observance of a relatively broad data set, which anyhow comprises various additional effects.

The criteria of the integral type are much easier for analytical evaluation of various load cases compared with the critical plane solution, by which the analytical definition of the plane with the maximum damage can get very cumbersome. To simplify the comparison, and to straighten its focus, each criterion is shown in Sec. 4 in its reduced variant involving only the axial stress amplitude and its mean value. The final criteria usable for a classic uniaxial load scenario are far from being simple in their formulation, and they cannot be in this way compared with the simplicity of e.g. the classic Goodman line. A question is raised and solved at the second part of the paper, if the additional material parameters involved in the multiaxial criteria give these criteria ability to cope with the mean stresses in a better way.

\section{Integral criteria analysed}

Most of the methods analysed here have been already evaluated in the extensive summary by Papuga [2]. The formulas of damage parameters of these methods are shown here in Table 1 for convenience. The dependencies of $a_{x}-d_{x}$ weight parameters of each $x$ method on the fatigue limits in basic uniaxial load conditions $\left(f_{-1}\right.$ for the fully reversed axial loading, $f_{0}$ for the case of repeated axial loading, $t_{-1}$ for the fully reversed torsion and $t_{0}$ for the repeated torsion) are described in Table 2.

Table 1. Formulas of all five integral criteria analysed in this paper. Abbreviations of methods' names used: FOG Fogue, LZ Liu \& Zenner, PAP Papadopoulos, PIN Papuga Integral method - new version, PIR Papuga Integral method as used in [2].

\begin{tabular}{|l|l|l|}
\hline $\begin{array}{l}\text { Abbrev. } \\
\text { [Ref] }\end{array}$ & General formula & \\
\hline FOG [6] & $\sqrt{\frac{1}{4 \pi} \int_{\varphi=0}^{2 \pi} \int_{\psi=0}^{\pi}\left(a_{F} C_{a}+b_{F} N_{a}+d_{F} N_{m}\right)^{2} \sin \psi d \psi d \varphi} \leq f_{-1}$ & (1) \\
\hline LZ [7] & $\sqrt{\left.\frac{15}{8 \pi} \int_{\varphi=0}^{2 \pi} \int_{\psi=0}^{\pi}\left[a_{L Z} C_{a}^{2}\left(1+c_{L Z} C_{m}^{2}\right)+b_{L Z} N_{a}^{2}\left(1+d_{L Z} N_{m}\right)\right)\right] \sin \psi d \psi d \varphi \leq f_{-1}}$ & (2) \\
\hline PAP [8] & $\sqrt{a_{p} \int_{\varphi=0}^{2 \pi} \int_{\psi=0}^{\pi} \int_{\chi=0}^{2 \pi}\left(T_{a}(\varphi, \psi, \chi)\right)^{2} d \chi \sin \psi d \psi d \varphi}+b_{p} \sigma_{H, \max } \leq f_{-1}$ & (3) \\
\hline PIN & $\sqrt{\frac{1}{4 \pi} \int_{\varphi=0}^{2 \pi} \int_{\psi=0}^{\pi}\left[a_{P N} C_{a}\left(C_{a}+c_{P N} C_{m}\right)+b_{P N}\left(N_{a}+d_{P N} N_{m}\right)\right] \sin \psi d \psi d \varphi} \leq f_{-1}$ & (4) \\
\hline PIR [2] & $\sqrt{\frac{1}{4 \pi} \int_{\varphi=0}^{2 \pi} \int_{\psi=0}^{\pi}\left[a_{P R} C_{a}^{2}+b_{P R}\left(N_{a}+d_{P R} N_{m}\right)\right] \sin \psi d \psi d \varphi} \leq f_{-1}$ & (5) \\
\hline
\end{tabular}

The weight parameters depicted in Table 2 are derived by analysing the integrals (1)-(5) for conditions of uniaxial loading (see [9] for a better detail). The pair of weights $a$ and $b$ can be retrieved from two uniaxial cases - (1) fully reversed axial loading and (2) fully reversed torsion. To define the parameters $c$ and $d$, two more basic load conditions are necessary repeated axial loading and repeated torsion. 
Table 2. All weight parameters of individual integral methods. Note that relevant indexes are used in Eqs. (1)-(11) to represent individual methods: F Fogue, LZ Liu-Zenner, P Papadopoulos, PN new PIN method, PR PIR method.

\begin{tabular}{|l|l|l|}
\hline Method & $\boldsymbol{a}$ parameter & $\boldsymbol{b}$ parameter \\
\hline FOG & $\sqrt{\frac{12 \cdot \kappa^{2}-21+b^{2}}{2}}$ & $\sqrt{\frac{15-3 \sqrt{25-8\left(\kappa^{2}-3\right)^{2}}}{2}}$ \\
\hline LZ & $\frac{1}{5} \cdot\left(3 \cdot \kappa^{2}-4\right)$ & $\frac{1}{5} \cdot\left(6-2 \cdot \kappa^{2}\right)$ \\
\hline PAP & $\frac{5}{8} \cdot(\kappa / \pi)^{2}$ & $3-\sqrt{3} \kappa$ \\
\hline PIN, PIR & $\frac{15}{2} \cdot \kappa \cdot\left(\frac{\pi \cdot \kappa-4}{3 \pi-4 \kappa}\right)$ & $3 \cdot f_{-1} \cdot\left[1-\kappa \cdot\left(\frac{\pi \cdot \kappa-4}{3 \pi-4 \kappa}\right)\right]$ \\
\hline Method & $\boldsymbol{c}$ parameter & $\boldsymbol{d}$ parameter \\
\hline FOG & $\frac{1}{3 a t_{0}^{2}}\left[\left(\frac{f_{-1}}{t_{0}}\right)^{2}-\frac{\kappa^{2}}{4}\right]$ & $\frac{28}{15 b f_{0}^{3}}\left[\left(\frac{2 f_{-1}}{f_{0}}\right)^{2}-\frac{a c f_{0}^{2}}{21}-1\right]$ \\
\hline LZ & $10 \frac{f_{-1}^{2}}{a t_{0}^{2}}-\frac{20}{3} \frac{b}{\pi a t_{0}}-1$ & $6 \frac{f_{-1}^{2}}{b f_{0}}\left(1-\frac{f_{0}^{2}}{3 t_{0}^{2}}\right)+\frac{4}{3} \frac{f_{0}}{\pi t_{0}}-1$ \\
\hline PIN & & $\kappa$ \\
\hline PIR & & $\kappa a)^{2}+45\left[4\left(\frac{f_{-1}}{f_{0}}\right)^{2}-1\right]-(3 b+2 a)$ \\
\hline
\end{tabular}

In addition to the weight parameters and fatigue limits, Table 2 contains one additional parameter - the fatigue limit ratio $\kappa$. It is the ratio between fatigue limits in fully reversed axial loading, and in fully reversed torsion. It is close to 1.0 for brittle materials, while most steels have $\kappa$ between 1.3 and 1.7. The formulations of $a$ and $b$ parameters in Table 2 shows some interesting properties. Fogue method cannot be used for materials with $\kappa$ below 1.111, otherwise $b$ parameter gets irrational. The $a$ weight parameter (and thus the effect of the shear stress) gets zero at $\mathrm{k}$ the square root of $4 / 3$ for the Fogue method and for the Liu-Zenner method, while both PIx methods meet this condition at $\kappa=4 / \pi$. All five methods discussed here reach the $b$ parameter zero at $\kappa$ being the square root of 3 .

\section{Full Benchmark}

\subsection{Description of the analysis}

The recent paper by Papuga et al. [5] states that the original database of experimental fatigue limits used for testing the multitude of methods in [2] was carefully re-validated, and though replenished by more new data, it got actually smaller than it used to be previously. This revised FatLim database of 286 items is used as the key data set for benchmarking the five integral methods. To analyse the quality of the prediction obtained by individual methods, the fatigue index error $\Delta F I$ is defined. The left-hand side of any formula in Eqs. (1) - (5) represents $\sigma_{e q}$ equivalent stress. The fatigue index error is then defined to be: 


$$
\Delta F I=\frac{\sigma_{e q}-f_{-1}}{f_{-1}}
$$

If the evaluated experimental data refer to the fatigue limit condition, the fatigue index error equals zero. If the criterion rates the equivalent stress higher than the fatigue limit, it leads to positive fatigue index error, which means it is conservative. Vice versa, negative values concern the non-conservative estimates. Due to the space requirements of this paper, only the final statistics of individual methods is presented in Table 3.

Table 3. The statistical analysis of the fatigue index error for five integral methods treated in this paper. To understand the measure of prediction quality, results of the PCN method of the critical plane type (see [3]) are added.

\begin{tabular}{|l|r|r|r|r|r|r|}
\hline & FOG & LZ & PAP & PIN & PIR & PCN \\
\hline $\max$ & $65.1 \%$ & $30.3 \%$ & $29.7 \%$ & $28.9 \%$ & $87.0 \%$ & $24.2 \%$ \\
\hline min & $-27.9 \%$ & $-54.6 \%$ & $-61.8 \%$ & $-15.4 \%$ & $-12.8 \%$ & $-22.4 \%$ \\
\hline range & $93.0 \%$ & $84.9 \%$ & $91.5 \%$ & $44.4 \%$ & $99.8 \%$ & $46.6 \%$ \\
\hline aver & $3.1 \%$ & $-1.2 \%$ & $-3.8 \%$ & $1.9 \%$ & $6.7 \%$ & $1.1 \%$ \\
\hline stdev & $15.5 \%$ & $9.2 \%$ & $14.5 \%$ & $7.2 \%$ & $18.1 \%$ & $6.1 \%$ \\
\hline
\end{tabular}

\subsection{Results}

The first glance at results gathered in Table 3 brings to the attention results of the PIR method. Its results are worse compared with other criteria presented here, and they are also worse compared with the original results present in [2]. The poor results obtained now are the outcome of two factors:

1. Change of the benchmark set, to which many new experimental data items were added, and some older experimental data items were revised.

2. The fact, that $d_{P R}$ weight parameter of the original PIR method [2] was tuned to fit the data items present there, without the adequate mathematical analysis, which would help to derive the mathematically correct weight of mean normal stress.

Though the benchmark set presented in [2] was larger than most previously used benchmark sets, it was not demanding enough to detect the poor behavior of the original PIR method. This is an important warning for any such analyses. It discloses the fact, that the range of data items used for further benchmarking should be continuously extended.

The summary in Table 3 covers the results of the PCN method introduced recently [3] in the last column. This is a method of the critical plane type, and its revision from the last PCR version [2] concerned also the implementation of the mean shear stress effect, similarly to the extension of original PIR to PIN. It is included in the analysis to provide some comparison of results of the integral methods with the method, which currently leads to the best possible results in the prediction quality.

Comparison of the PCN method with the new PIN results in the conclusion, that PIN has a very similar range of results, but the scatter of results manifested in the parameter of the standard deviation is clearly worse. From integral methods anyhow, the PIN methods stays on top as regards the evaluation of the prediction quality. The LZ method, which has the same number of material parameters (4) gets worse both in the range and in the standard deviation. The Fogue method and the Papadopoulos method are substantially worse, with the standard deviation being twice of the standard deviation obtained by the PIN method. The similar level of the overall prediction quality of these two methods is interesting, because the Fogue method relies on three material parameters compared with just two of them by the Papadopoulos method - the addition of the third material parameter did not help at all. 
The comparison of the overall prediction quality brings along only a little understanding, which effects could potentially induce worsening of the prediction quality of any inspected criterion. Table 4 is therefore prepared as a minimum tool to cope with this problem, while keeping the paper within the required length. The groups are chosen so that the influence of the mean stress on various load channels could be traced.

The effect of compressive mean stress is analyzed separately, because the opinion that it should be specially treated in the formulation of the equivalent stress amplitude can be found (see [10]). Except for the Fogue method, which goes in the opposite trend, other methods tend to get more conservative in the mean value of $\Delta F I$, if the mean stress shifts to the compressive domain, and the standard deviation is not substantially changed.

The Fogue method apparently suffers from the inadequate phase shift effect, which manifests itself above all in the nMS,OP group. Its composition relying only on the mean normal stress and neglecting the mean shear stress is an additional reason for bad results and the comparison of Ax and To groups in the mean value of $\triangle F I$ proves that well.

The Papadopoulos method is based on just two material parameters. The mean stress effect is assumed to be covered by including maximum hydrostatic stress. Varying results of the mean $\Delta F I$ value in different groups, with the peak of the poor performance in the groups Ax and To, manifest clearly that the mean stress effect is not mastered sufficiently.

The Liu-Zenner method and the PIN method, which both use two additional material parameters to cover the mean stress effect are apparently producing the best results within the given groups. The stability of the mean $\triangle F I$ value in the LZ method is astonishing, but the PIN method clearly shows markedly lower scatter - the LZ method does not have a better standard deviation in any of groups depicted in Table 4.

Table 4. The statistical analysis of $\Delta F I$ for integral methods, if various groups are formed from the benchmark set. Abbrevitions: !negMS - there are no negative mean stresses, negMS - only load cases with negative mean stress, MS - only load cases including at least one mean stress, nMS - only load cases without any mean stress, OP - out-of-phase loading, Ax - only axial loading, To - only torsion.

\begin{tabular}{|l|l|l|l|l|l|l|l|l|l|l|l|}
\hline \multirow{2}{*}{ Group } & \multirow{3}{*}{ Items } & \multicolumn{4}{|c|}{ Average $\Delta$ FI [\%] } & \multicolumn{5}{c|}{ Standard deviation $\Delta$ FI [\%] } \\
\cline { 3 - 12 } & & FOG & LZ & PAP & PIN & PIR & FOG & LZ & PAP & PIN & PIR \\
\hline All & 286 & 3.1 & -1.2 & -3.8 & 1.9 & 2.7 & 15.5 & 9.2 & 14.5 & 7.2 & 10.5 \\
\hline !negMS & 254 & 3.4 & -1.5 & -4.2 & 1.3 & 2.1 & 15.8 & 8.5 & 14.6 & 7.0 & 10.4 \\
\hline negMS & 30 & 0.6 & 3.6 & -0.5 & 6.7 & 7.8 & 12.9 & 8.5 & 14.0 & 7.7 & 10.3 \\
\hline MS & 196 & 1.1 & -1.2 & -6.8 & 2.7 & 4.1 & 12.8 & 10.1 & 16.1 & 7.7 & 11.9 \\
\hline nMS & 90 & 7.3 & -1.0 & 2.8 & 0.2 & -0.2 & 19.5 & 6.7 & 6.7 & 5.6 & 5.8 \\
\hline nMS,OP & 29 & 11.6 & -1.3 & 5.3 & 1.0 & 0.4 & 22.7 & 8.4 & 9.3 & 5.5 & 5.7 \\
\hline Ax & 39 & 6.9 & -1.2 & -14.0 & 3.1 & 6.7 & 16.1 & 7.5 & 19.4 & 6.9 & 18.1 \\
\hline To & 19 & -7.9 & 1.2 & -17.8 & -2.2 & -4.8 & 12.1 & 8.7 & 12.2 & 5.7 & 7.5 \\
\hline
\end{tabular}

\section{Benchmark on axial loading}

\subsection{Multiaxial criteria}

The conclusion from the last section is clear - the most effective in the good estimate of the equivalent stress are criteria LZ and PIN, which are both based on four material parameters, two of which relate to repeated load modes. The stability of the response of both criteria is good over different groups of load modes. The criteria in their complex integral formulation can be reduced for purely axial loading to simpler formula without integrals - see Table 5 . 
Table 5. Reduction of the original formulations in Eqs. (1) - (5) for the cases of purely axial loading.

\begin{tabular}{|l|l|l|}
\hline Method & Formula for axial stress amplitude $\sigma_{a}$ and axial mean stress $\sigma_{\boldsymbol{m}}$ & \\
\hline FOG & $\sqrt{\frac{2}{15}\left(a_{F}^{2} \sigma_{a}^{2}+2 a_{F} b_{F} \sigma_{a}^{2}+2 a_{F} d_{F} \sigma_{a} \sigma_{m}\right)+\frac{1}{5}\left(b_{F} \sigma_{a}+d_{F} \sigma_{m}\right)^{2}} \leq f_{-1}$ & (7) \\
\hline $\mathrm{LZ}$ & $\sqrt{\frac{4}{21} \cdot a_{L Z} \cdot c_{L Z} \cdot \sigma_{a}^{2} \cdot \sigma_{m}^{2}+\frac{15}{14} \cdot b_{L Z} \cdot d_{L Z} \cdot \sigma_{a}^{2} \cdot \sigma_{m}+\sigma_{a}^{2} \leq f_{-1}}$ & (8) \\
\hline $\mathrm{PAP}$ & $\sigma_{a}+\sigma_{m} \cdot\left(1-\frac{f_{-1}}{\sqrt{3} \cdot t_{-1}}\right) \leq f_{-1}$ & (9) \\
\hline $\mathrm{PIN}$ & $\sqrt{\frac{2}{15} \cdot a_{P N} \cdot \sigma_{a} \cdot\left(\sigma_{a}+c_{P N} \cdot \sigma_{m}\right)+\frac{1}{3} \cdot b_{P N} \cdot\left(\sigma_{a}+d_{P N} \cdot \sigma_{m}\right)} \leq f_{-1}$ & $(10)$ \\
\hline $\mathrm{PIR}$ & $\sqrt{\frac{2}{15} \cdot a_{P R} \cdot \sigma_{a}^{2}+\frac{1}{3} \cdot b_{P R} \cdot\left(\sigma_{a}+d_{P R} \cdot \sigma_{m}\right)} \leq f_{-1}$ & $(11)$ \\
\hline
\end{tabular}

The hope, that this reduction enabled by integrating a simpler load cases could also reduce the complexity of the criterion as regards the number of material parameters that affect its response, is not meeting the reality. The number of material parameters involved in the criteria in Eqs. (7) - (11) stays the same as it is in the general configuration in Eqs. (1) - (5). This means that all criteria assume that torsion properties of the material affect also the way the material responds under purely axial loading. Existence of such dependency should be observable, if only axial load cases are analysed. According to Table 4, there are 39 such data items in the whole benchmark. These test data thus will be used for further analyses including the comparison with uniaxial methods for the mean stress effect analysis.

\subsection{Uniaxial criteria}

There is a multiple of methods focused on the mean stress effect analysis in the high-cycle fatigue, see the survey in [11-12]. The best-known Goodman and Gerber methods are based on only one additional material parameter - the tensile strength $S_{u}$. The general experience from [11] is that the use of these two methods results in quite poor prediction results. If some replacement similar with the Goodman method

$$
\frac{\sigma_{a}}{1-\frac{\sigma_{m}}{S_{u}}} \leq f_{-1}
$$

has to be proposed, the Half-slope method [11] is a more accurate proposal:

$$
\frac{\sigma_{a}}{1-\frac{\sigma_{m}}{2 \cdot S_{u}}} \leq f_{-1}
$$

The paper [11] comments that the poor output of these criteria is partly caused by the fact that the experimental data include the cases of push-pull and also of bending. The Goodman method is not good for such cases above all, because the tensile strength can be exceeded in elastic stress values at the outmost layers of bended specimens. 
The best performance as regards the prediction quality was found for two methods derived from the SWT (Smith, Watson, Topper) criterion. The Bergmann method states:

$$
\sqrt{\sigma_{a} \cdot\left(\sigma_{a}+k_{B} \cdot \sigma_{m}\right)} \leq f_{-1}
$$

while the Walker method formula is:

$$
\sigma_{a}^{w} \cdot\left(\sigma_{a}+\sigma_{m}\right)^{1-w} \leq f_{-1}
$$

For the whole benchmark set, the values of the fatigue strength in repeated loading is ready for the use as the important material parameters. It is thus reasonable to use it (similarly to the multiaxial methods) as the primary material parameter to derive the coefficients $k_{B}$ and $w$ of both methods. In the case of the Bergmann method, the parameter can be obtained from:

$$
k_{B}=\left(\frac{2 \cdot f_{-1}}{f_{0}}\right)^{2}-1,
$$

while the use of the Walker method for this load case results in:

$$
w=\frac{\log \frac{f_{-1}}{f_{0}}}{\log 2} .
$$

The same type of prediction quality evaluation by the $\Delta F I$ parameter (see Eq. (6)) can be used here, because the left-hand sides of Eqs. (14) and (15) correspond to equivalent stress. To better reflect the response to mean stress, three groups of mean stress (MS) levels were established:

- $\quad$ MS negative - Though there are only 4 such items in the benchmark set, various criteria are not able to cope with that without some further interference with their formulation. The Walker method collapses to $\sigma_{e q}=0$ for repeated compression loading $(R=-\infty$; one of this load case in the benchmark set could not be thus evaluated by the Walker methods), and leads to irreal numbers if the mean stress goes further past this point to higher compression. The Bergmann method leads to the same problems for $\sigma_{m}<-\sigma_{a} / k_{B}$ (this condition has not been met here).

- MS low - Most criteria evaluated here (with the exception of the Papadopoulos method and the Goodman and Half slope methods) are tuned to optimally respond to the load cases of fully reversed axial loading $(R=-1)$ and of repeated axial loading $(R=0)$. Prediction for these two load cases thus will be perfect for these methods, and it can be anticipated, that also the space in between them (mild mean stresses) will be covered quite well, with little differences in the quality of individual methods. This group is defined by the upper limit $\sigma_{m} / \sigma_{a}<1.2$.

- MS high - This group covers all cases $\sigma_{m} / \sigma_{a}>1.2$.

The analyses in [10] show that the Goodman method is not suitable for plane bending cases. This is the reason why these load cases were separated into one group (PB), while the tensile load cases (Ten) to another group. Indeed, the comparison of the mean and standard deviation value in Tables 6 and 7 confirms that conclusion. The Half-slope method is much more successful if only these two methods, in which the mean stress effect is related to the tensile strength, are compared. Anyhow, both methods are the worst ones in the complete set of methods, including the multiaxial methods.

The Walker method and the Bergmann method provide much better response, which only two multiaxial methods (LZ and PIN) can exceed if only the complete set is evaluated. The most marked differences can be found in the group MS high, with high mean stresses involved. The PIN method gets too conservative there in mean, and both multiaxial methods 
result in a higher scatter of the fatigue index error than the Bergmann method does. On the other hand, PIN method provides much better response to the compressive mean stresses, for which both uniaxial methods fail to respond well.

The group of negative mean stresses has only four data items, thus this finding is not supported well enough. More such experimental data items are necessary. Results for these load cases largely affect the otherwise superior response of the Bergmann method. If this group is omitted, it does not seem that involving the torsion fatigue parameters into the mean stress effect analysis brings along some improvement of the prediction quality compared with methods, which use only the axial stress response. On the other hand, the question, if the torsion properties can help to understand or to describe the response of the material under compression mean stresses, can be raised and could be further addressed in a next research.

Table 6. Average $\triangle F I$ results for individual methods. New abbreviations: $\mathrm{W}-$ Walker method, $\mathrm{B}-$ Bergmann method, G - Goodman method, HS - Half slope method.

\begin{tabular}{|l|l|l|l|l|l|l|l|l|l|l|}
\hline Group & Items & FOG & LZ & PAP & PIN & PIR & W & B & G & HS \\
\hline All & 39 & 6.9 & -1.2 & -14.0 & 3.1 & 6.7 & 0.9 & -1.7 & -61.7 & -5.7 \\
\hline MS low & 17 & 4.2 & -0.2 & -9.5 & 0.2 & 3.0 & 0.5 & 0.3 & 23.8 & -2.1 \\
\hline MS high & 18 & 12.9 & -1.0 & -21.4 & 6.2 & 10.7 & 3.7 & 1.8 & -154.3 & -11.1 \\
\hline MS neg. & 4 & -8.4 & -5.9 & 0.6 & 1.5 & 4.6 & -9.7 & -26.2 & -7.7 & 3.7 \\
\hline Ten & 22 & 5.6 & -2.0 & -13.2 & 4.7 & 8.7 & 1.9 & -3.2 & 20.2 & -15.6 \\
\hline PB & 17 & 8.6 & -0.1 & -15.0 & 1.1 & 4.2 & -0.3 & 0.2 & -167.6 & 7.2 \\
\hline
\end{tabular}

Table 7. The standard deviations of $\Delta F I$ results for individual methods.

\begin{tabular}{|l|l|l|l|l|l|l|l|l|l|l|}
\hline Group & Items & FOG & LZ & PAP & PIN & PIR & W & B & G & HS \\
\hline All & 39 & 16.1 & 7.5 & 19.4 & 6.9 & 18.1 & 10.5 & 11.1 & 709.9 & 27.3 \\
\hline MS low & 17 & 12.3 & 2.6 & 10.3 & 1.2 & 15.5 & 1.7 & 1.5 & 38.1 & 15.4 \\
\hline MS high & 18 & 17.6 & 10.1 & 24.2 & 9.0 & 21.6 & 14.1 & 8.2 & 1052.6 & 35.8 \\
\hline MS neg. & 4 & 11.5 & 7.8 & 12.3 & 6.1 & 5.7 & 7.7 & 16.1 & 22.5 & 24.2 \\
\hline Ten & 22 & 17.3 & 9.7 & 20.9 & 8.7 & 20.0 & 13.9 & 14.6 & 38.7 & 21.7 \\
\hline PB & 17 & 14.6 & 2.8 & 17.9 & 2.8 & 15.5 & 2.6 & 2.0 & 1083.4 & 29.1 \\
\hline
\end{tabular}

\section{Conclusion}

The paper covers the topic of the mean shear stress effect, which was implemented into the older PIR criterion [2]. The new criterion PIN was assessed together with other integral multiaxial fatigue strength criteria (by Fogue, by Liu and Zenner, by Papadopoulos) on a large benchmark set of 286 fatigue strengths within the HCF domain. The results of the new PIN variant are substantially better, than are the results of the original PIR criterion. Overall, results of the PIN method are better than those obtained by other integral methods.

The reduction of the original integral formula for the case of purely axial loading was done, and the final formulas were presented. All of them keep the same numbers of necessary input material parameters as they were in original integral formulations. A test was done, whether the inclusion of torsion parameters intrinsically present in these formulations helps to provide better prediction results under purely axial loading. The comparison with more sophisticated uniaxial mean stress effect formulations (by Walker or by Bergmann) is done 
on the smaller benchmark set of 39 items. The result is that the Bergmann method is slightly better than PIN in the prediction under purely axial loading with high mean stresses, but it fails in the case of compressive mean stresses. Questions, whether the superior behaviour of the PIN method in the compressive region is real and whether this could be related to the presence of torsion properties, remain open, because this group of experiments concerned only 4 items.

Authors acknowledge support from the ESIF, EU Operational Programme Research, Development and Education, and from the Center of Advanced Aerospace Technology (CZ.02.1.01/0.0/0.0/16_019/0000826), Faculty of Mechanical Engineering, Czech Technical University in Prague. Further support for the research was provided within the project from the Grant Agency of the Czech Technical University in Prague (grant no. SGS17/175/OHK2/3T/12).

\section{References}

1. J. Papuga: Improvements of two criteria for multiaxial fatigue limit evaluation. Bull of Appl Mech, 5: 80-86 (2009)

2. J. Papuga: A survey on evaluating the fatigue limit under multiaxial loading. Int Jnl of Fatigue, 33: 153-165 (2011)

3. J. Papuga; R. Halama: Mean stress effect in multiaxial fatigue limit criteria. Arch. Appl. Mech. (2019), https://doi.org/10.1007/s00419-018-1421-7.

4. J. Tomčala, J. Papuga, D. Horák, V. Hapla, M. Pecha, M. Čermák: Steps to increase practical applicability of PragTic software, 129: 57-68 (2019)

5. J. Papuga, I. Vízková, M. Nesládek, Š. Trubelová: Validation data set for testing the criteria for multiaxial fatigue strength estimation. Fat \& Fract of Engng Mat \& Structs, 41: 2259-2271 (2018)

6. M. Fogue: Critére de fatigue a longue durée de vie pour des états multiaxiaux de contraintes sinusoidales en phase et hors phase. [PhD thesis]. Lyon, INSA de Lyon 1987.

7. H. Zenner, A. Simbürger, J. Liu: On the fatigue limit of ductile metals under complex multiaxial loading. Int Jnl of Fatigue, 22: 137-145 (2000)

8. I.V. Papadopoulos, P. Davoli, C. Gorla, M. Filippini, A. Bernasconi: A comparative study of multiaxial high-cycle fatigue criteria for metals. Int Jnl of Fatigue 19: 219-235 (1997)

9. J. Papuga, M. Růžička: Two new multiaxial criteria for high cycle fatigue computation. Int Jnl of Fatigue, 30: 58-66 (2008)

10. B. Kenmeugne, B. D. Soh Fotsing, M. Fogue, G. Kuate, J.-L. Robert: More accurate taking into account of the large compressive mean stresses in fatigue criteria by a threshold approach. Int Jnl of Adv Engng Sci \& Tech 7: 122-132 (2011).

11. J. Papuga, I. Vízková, M. Lutovinov, M. Nesládek: Mean stress effect in stress-life fatigue prediction re-evaluated. In: Hénaff, G. (ed.) MATEC Web of Conferences, 165 (2018)

12. J. Papuga, I. Vízková, M. Lutovinov, M. Nesládek: Comparison of Several Optimized Methods for Mean Stress Effect Evaluating the Stress-Life Prediction. In: Mechanical Fatigue of Metals - Experimental and Simulation Perspectives, Springer International Publishing (2019). 